\title{
Muscle Metastases Revealing Gastric Signet Ring Cell Carcinoma: Case Report and Review of the Literature
}

\author{
Mokhlis MA ${ }^{1 *}$, Tanz $\mathbf{R}^{1}$, Mahfoud T', Khmamouch \\ $\mathbf{R}^{1}$, Debbagh $A^{1}$, Errihani $\mathbf{H}^{2}$ and Ichou $\mathbf{M}^{1}$ \\ ${ }^{1}$ Department of Medical Oncology, Military Hospital, \\ Mohamed V, Rabat, Morocco \\ ${ }^{2}$ Department of Medical Oncology, National Institute of \\ Oncology, Rabat, Morocco \\ *Corresponding author: Mohamed Anouar Mokhlis, \\ Department of Medical Oncology, Military Hospital, \\ Mohamed V, Rabat, Morocco
}

Received: December 10, 2018; Accepted: J anuary 08, 2019; Published: January 15, 2019

\begin{abstract}
Advanced stomach cancer remains a serious disease with a poor prognosis. Skeletal muscle metastases of gastric carcinoma are extremely rare and may be revealing the disease. The signet ring cell carcinoma is defined by the presence of proportion of independent cells greater than $50 \%$. In an advanced stage it has a more aggressive character with a greater risk of lymph node invasion or distant metastasis. The needle biopsy is required for diagnosis. The chemoresistance of SRCC appear as elements of poor prognosis. The armamentarium includes further the antitumor chemotherapy, radiotherapy and palliative care adapted to the patient's clinical context.
\end{abstract}

Keywords: Muscle Metastasis; Signet Cell Carcinoma; Gastric Cancer; Chemoresistance

\section{Abbreviations}

SRCC: Signet Ring Cell Carcinom; CT: Computer Tomography; MRI: Magnetic Resonance Imaging; FDG -PET: Fluorodeoxyglucose (FDG)-Positron Emission Tomography; FOLFOX: 5-Fluoro-Uracile, Leucovorin and Oxaliplatine; FOLFIRI: 5-Fluoro-Uracile, Leucovorin and Irinotecan

\section{Introduction}

Gastric cancer is the second most common digestive cancer after colorectal cancer and the fourth leading cause of cancer death worldwide [1].

The most common metastatic sites are regional lymph nodes, liver, peritoneum, lung and bone. Gastric cancer can cause unusual metastases, such as gums, irises, testicles, muscles or even the meninges [2,3]. Muscular metastases are rare, only sporadic cases have been reported in the literature $[4,5]$.

We report, in one patient, the case of a gastric adenocarcinoma with signet ring cells, which was revealed by the presence of muscular metastases.

\section{Case Presentation}

This is a 54-year-old Caucasian woman with a history of arterial hypertension who was admitted in July 2018 to manage a painful abdominal swelling affecting the right flank evolving for 2 months in a context of paroxysmal epigastralgia. On admission, she was $160 \mathrm{~cm}$ tall and weighed $65 \mathrm{~kg}$. She had a temperature of $36.4^{\circ} \mathrm{C}$, heart rate 82 beats $/ \mathrm{min}$, blood pressure $140 / 70 \mathrm{mmHg}$. The abdominal examination found a hard and sensitive mass of the right flank, which was fixed in relation to the deep plane, the ganglionic areas were free, and the rest of the examination was without particularity.

Therefore, she received abdominal pelvic CT demonstrating irregular nodular thickening of the right transverse and the right rectus muscles of the abdomen with edematous diffuse infiltration of the next subcutaneous fatty tissue and a diffuse thickening of the cardiotuberotic and fundic region of $2 \mathrm{~cm}$ thick (Figure 1). Subsequently, an ultrasound-guided biopsy of muscle swelling was conducted. The histological result with immunohistochemical complement (anti-CK7 +, anti-CK20 +, WT1-, ACE +, CDX2 + antibody) was in favor of muscle metastasis of gastric signet ring cell carcinoma (Figure 2 and 3). Endo-gastroduodenal fibroscopy showed a non-stenosing ulcerobourgonnant antral process. The histological diagnosis of the gastric biopsy showed a signet ring cell carcinoma with a background of chronic gastritis HP- and the HER2 status was negative.

At this stage, a full-body CT with contrast and PET-scan (Figure 4) had excluded other distant metastases from gastric carcinoma, and the ACE/CA19-9 tumor markers were negative.

After a multidisciplinary evaluation, considering the advanced stage of the disease (stage IV) and the absence of gastrointestinal complications, including gastrointestinal bleeding or gastric stenosis, a modified FOLFOX-4 regimen (J1-J15) was administered as firstline chemotherapy. After 3 cycles, the clinical and radiological evaluation by thoraco-abdominopelvic CT showed a reduction

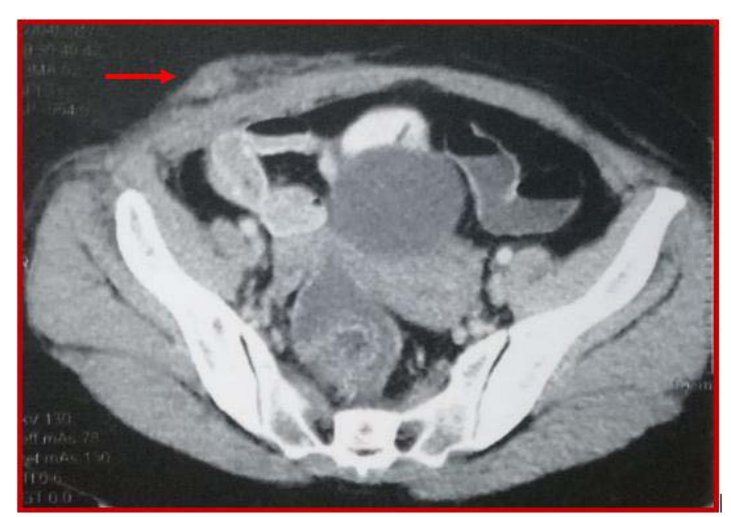

Figure 1: Abdominal pelvic CT shows irregular nodular thickening of the right transverse and the right rectus muscles of the abdomen.
Austin J Clin Case Rep - Volume 6 Issue 1 - 2019 ISSN : 2381-912X | www.austinpublishing group.com Mokhlis et al. (C) All rights are reserved
Citation: Mokhlis MA, Tanz R, Mahfoud T, Khmamouch R, Debbagh A, Errihani H, et al. Muscle Metastases Revealing Gastric Signet Ring Cell Carcinoma: Case Report and Review of the Literature. Austin J Clin Case Rep. 2019; 6(1): 1137. 


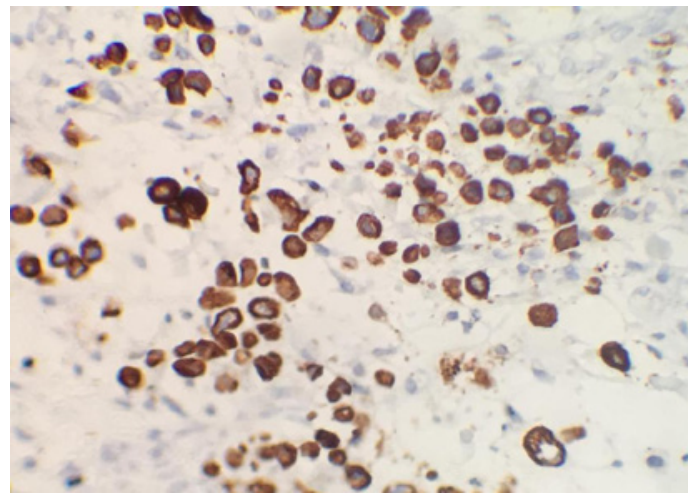

Figure 2: Higher magnification (x200) reveals poorly differenciated signet ring cell carcinoma composed of single, non-cohesive, round and vacuolated cells.

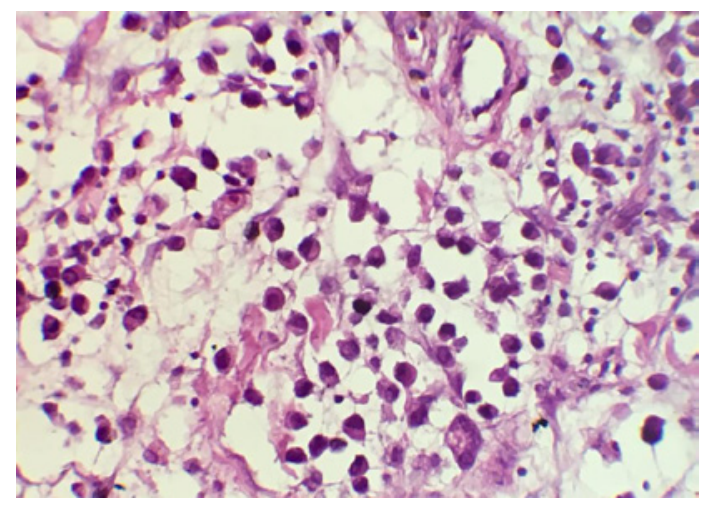

Figure 3: Immunohistochemical complement; Microphotograph showing intracellular mucin within tumor cells. Nuclear atypia and mitosis are also visible. [PAS, $\times 400]$.

in gastric thickening and metastatic muscle thickening. The consultation with the surgeons did not retain the indication of carcinologic excision. After receiving an additional cycle, the patient objectified the appearance of a rapidly progressive swelling affecting the left flank and the left iliac fossa (Figure 5) and the appearance of a left vulvar swelling. A lesion progression was also retained on the Thoraco-Abdomino-Pelvic CT data showing the appearance of a new lesion in the left rectus muscle (Figure 6) and on the vulvar biopsy data confirming a secondary localization of a signet ring cell carcinomatous process compatible with known gastric origin (AC antiparcytokeratin + ).

Considering the conservation of the patient performance status, a second-line chemotherapy was selected based on FOLFIRI regimen (J1-J15). The patient is currently under treatment.

\section{Discussion}

Despite recent advances in cancer therapy, advanced stomach cancer remains a serious disease with a poor prognosis. It is an adenocarcinoma in $95 \%$ of cases and, in 20\% of cases, it is accompanied by overexpression of the HER2 receptor. The signet ring cell carcinoma (SRCC) is defined by the presence of proportion of independent cells (weakly cohesive cells or also called ring-shaped cells) greater than $50 \%$.

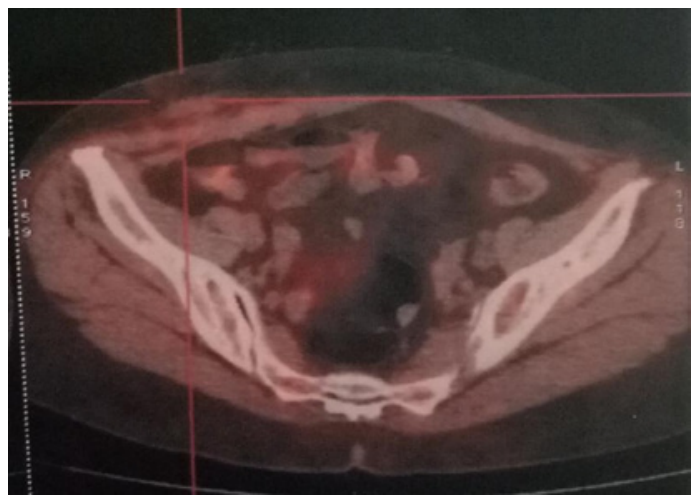

Figure 4: FDG-PET SCAN image exclude other distant metastases from gastric carcinoma.

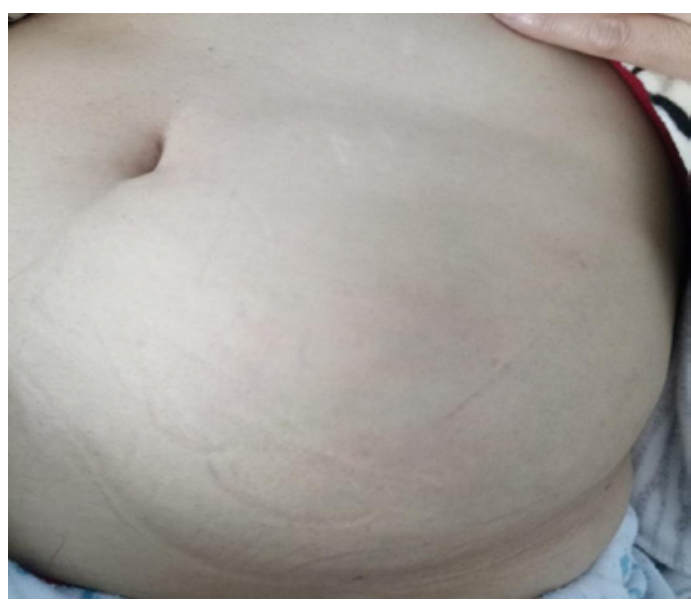

Figure 5: Rapidly progressive swelling affecting the left flank and the left iliac fossa.

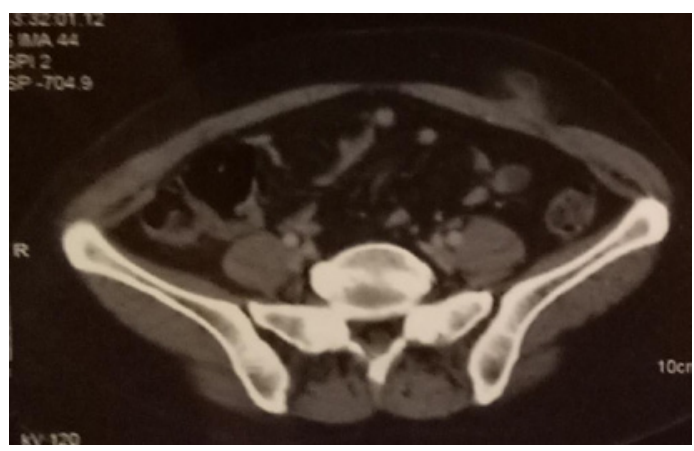

Figure 6: Thoraco-Abdomino-Pelvic CT data shows the appearance of a new lesion in the left rectus muscle.

SRCC appears as a separate entity affecting more often women and at a younger age (median age at diagnosis: 61.9 years) [5] and preferentially sits in the antrum and body of the stomach. In addition, it may be related to a genetic predisposition in carriers of the $\mathrm{CDH} 1$ gene mutation encoding E-cadherin. At an early stage, SRCC is a type of gastric cancer with a similar prognosis as the rest of gastric carcinoma subtypes, but in an advanced stage it has a more aggressive character with a greater risk of lymph node invasion or distant 
metastasis. The presence of muscle metastases is rarely described in the literature [5]. The primary cancers commonly associated with muscle metastases are lung, kidney, bladder cancers, and carcinomas of the gastrointestinal tract. The anatomical predilection sites for occurrence of muscle metastases are the lower limbs [6,7]. In the literature, cases that associate gastric cancer with muscle metastases are rare, and most of the cases described have a histological profile of SRCC and are associated with advanced disease and poor prognosis. Several other unusual sites of metastasis of gastric cancer have been reported in the literature such as pancreatic, cutaneous, parotid, gingival, mammary, testicular, uterine and fallopian tube metastasis $[8,9]$.

To date, although skeletal muscle is a hypervascularized tissue and constitutes more than half of the body mass, there are no clear explanations justifying the scarcity of muscle metastases. Several factors could make skeletal muscle hostile to metastatic spread, such as unconstant muscle blood flow, pressure changes due to muscle contractions, inhibition of tumor proliferation by lactic acid, muscle $\mathrm{pH}$, and portal filter that can prevent the peripheral spread of the disease apart from the presence of hepatic metastasis [10-12].

Muscular metastases can be discovered incidentally during a radiological assessment in asymptomatic patients or more generally by the presence of a hard and painful mass $[13,14]$. They may also be indicative of an undiagnosed primary tumor, as in our case, with or without other detectable metastases $[4,5,7,15]$.

Although most skeletal muscle metastases are detected by CT, MRI is considered superior to CT in terms of characterization of muscle abnormalities $[7,8,14]$.

When the skeletal muscle metastasis is the initial presentation, as in our patient, the biopsy is considered obligatory for diagnosis as long as the mass is accessible $[6,13]$.

The medical treatment of advanced gastric cancers is essentially based on chemotherapy, to which can be added other therapeutic options including radiotherapy, to relieve pain or to reduce the size of muscular metastases, and surgical excision which can be proposed in selected patients with a symptomatic isolated mass in the absence of other metastatic locations [6,7]. However, several SRCC data from gastric adenocarcinoma studies suggest chemoresistance in this histological subtype $[16,17]$.

Metastatic SRCC have a poor prognosis and depend mainly on metastatic spread. In our case, rapid progression with the appearance of other sites of muscle metastasis after only four cycles of chemotherapy, even in the absence of visceral metastases, made surgical excision or other local treatment impossible.

\section{Conclusion}

Skeletal muscle metastases of gastric carcinoma are extremely rare and may be revealing the disease. As in our case, the needle biopsy is required for diagnosis. In addition, any painful tumefaction must make suspect the presence of muscular metastases. These as well as the chemoresistance of SRCC appear as elements of poor prognosis. The armamentarium includes further the antitumor chemotherapy, radiotherapy and palliative care adapted to the patient's clinical context.

\section{References}

1. Ferlay J, Shin HR, Bray F, Forman D, Mathers C, Parkin DM. Estimates of worldwide burden of cancer in 2008: GLOBOCAN 200. Int J Cancer. 2010; 127: 2893-2917.

2. Shimoyama S, Seto Y, Aoki F, et al. Gastric cancer with metastasis to the gingiva. J Gastroenterol Hepatol. 2004; 19: 831-835.

3. Bulut G, Erden A, Karaca B, et al. Leptomeningeal carcinomatosis of gastric adenocarcinoma. Turk J Gastroenterol. 2011; 22: 195-198.

4. Pestalozzi BC, von Hochstetter AR. Muscle metastasis as initial manifestation of adenocarcinoma of the stomach. Schweiz Med Wochenschr. 1998; 128 : 1414-1417.

5. Rosenbaum LH, Nicholas JJ, Slasky BS, et al. Malignant myositis ossificans: occult gastric carcinoma presenting as an acute rheumatic disorder. Am Rheum Dis. 1984; 43: 95-97.

6. Herring CL Jr, Harrelson JM, Scully SP. Metastatic carcinoma to skel.0000etal muscle. A report of 15 patients. Clin Orthop Relat Res. 1998; 355: 272-281.

7. Tuoheti $\mathrm{Y}$, Okada K, Osanai $\mathrm{T}$, et al. Skeletal muscle metastases of carcinoma: a clinicopathological study of 12 cases. Jpn J Clin Oncol. 2004; 34: $210-214$.

8. Wente MN, Bergmann F, Fröhlich BE, et al. Pancreatic metastasis from gastric carcinoma: a case report. World J Surg Oncol. 2004; 2: 43.

9. Luk YS, Ka SY, Lo SS, et al. An unusual case of gastric cancer presenting with breast metastasis with pleomorphic microcalcifications. J Breast Cancer. 2012; 15: 356-358.

10. Seely S. Possible reasons for the high resistance of muscle to cancer. Med Hypotheses. 1980; 6: 133-137.

11. Weiss L. Biomechanical destruction of cancer cells in skeletal muscle: a rate-regulator for hematogenous metastasis. Clin Exp Metastasis. 1989; 7: 483-491.

12. Namba T, Sato T, Grob D. Inhibition of Ehrlich ascites tumors cells by skeleta muscle extracts. Br J Exp Pathol. 1968; 49: 294-301.

13. Tougeron $\mathrm{D}$, Hamidou $\mathrm{H}$, Dujardin $\mathrm{F}$, et al. Unusual skeletal muscle metastasis from gastric adenocarcinoma. Gastroenterol Clin Biol. 2009; 33: 485-487.

14. Arpaci T, Ugurluer G, Akbas T, et al. Imaging of the skeletal muscle metastases. Eur Rev Med Pharmacol Sci. 2012; 16: 2057-2063.

15. Oba K, Ito T, Nakatani C, et al. An elderly patient with gastric carcinoma developing multiple metastasis in skeletal muscle. J Nippon Med Sch. 2001; 68: $271-274$.

16. Rougier P, Ducreux M, Mahjoubi M, Pignon JP, Bellefqih S, Oliveira J, et al. Efficacy of combined 5-fluorouracil and cisplatinum in advanced gastric carcinomas. A phase II trial with prognostic factor analysis. Eur J Cancer. 1994: 30A: 1263-1269.

17. Takiuchi H, Hirata I, Kawabe S, Egashira Y, Katsu K. Immunohistochemical expression of vascular endothelial growth factor can predict response to 5 -fluorouracil and cisplatin in patients with gastric adenocarcinoma. Oncol Rep. 2000; 7: 841-846. 